\title{
ON GENERATING SYSTEMS FOR ABELIAN GROUPS
}

\section{P. L. SPERRY}

The purpose of this note is to investigate two kinds of generating sets for an abelian group. It is well known that if an abelian group has a projective cover then it has a very special kind of generating set: a free basis. The idea of a projective cover of an abelian group is dual to that of a divisible hull when the divisible hull is defined as an essential injective extension. This note considers the dualization of two other equivalent definitions of a divisible hull. This yields the definitions of a $q$-cover and an $i$-cover. It is shown that an abelian group has a $q$-cover if and only if it has a minimum system of generators (in the sense of Khabbaz). It is also shown that the only torsion free abelian groups which have $q$-covers are the free groups and that the torsion subgroup of an abelian group has a $q$-cover if the group does. These two facts give rise to the suspicion that there is a direct connection between groups with $q$-covers and splitting groups; two examples are given which show that this suspicion is false. Finally it is shown that very many abelian groups have $i$-covers; included among these are all torsion free groups and all groups which have $q$-covers.

Throughout this note all groups are abelian and the notation, for the most part, follows [1]. In particular: $r(G)$ is the rank of $G, r^{*}(G)$ is the reduced rank of $G, G_{t}$ is the torsion subgroup of $G, Z$ is the additive group of integers and $Z_{n}$ is the integers $\bmod n$. A subgroup $H$ of $G$ is small in $G$ if for any subgroup $K$ of $G,\{H, K\}=G$ implies $K=G$.

Definition (Khabbaz [3]). A subset $S$ of a group $G$ is a minimum system of generators (abbreviated: M.s.g.) for $G$ if $\{S\}=G$ and no finite subset $S_{1}$ of $S$ may be replaced by a smaller subset $T$ of $G$ in such a way that $\left\{\left(S-S_{1}\right), T\right\}=G$.

Definition. A $q$-cover for a group $G$ is a free group $F$ together with an epimorphism $\phi$ of $F$ onto $G$ such that for any proper direct summand $F_{1}$ of $F, \phi\left[F_{1}\right] \neq G$.

An $i^{*}$-cover of $G$ is a free group $F$ together with an epimorphism $\phi$ of $F$ onto $G$ such that if $F_{1}$ is free, $\psi$ an epimorphism of $F_{1}$ onto $G$ and $\sigma$ is a homomorphism of $F$ into $F_{1}$ such that $\psi \sigma=\phi$ then $\sigma$ is a monomorphism.

Received by the editors March 10, 1969. 
THEOREM 1. The following are equivalent:

(I) G has a q-cover.

(II) $G$ has a generating set $S$ such that any finite subset of $S$ is a M.s.g. for the subgroup it generates.

(III) There is a free group $F$ and an epimorphism $\phi$ of $F$ onto $G$ such that ker $\phi$ contains no nonzero direct summand of $F$.

(IV) G has an $i^{*}$-cover.

(V) G has a M.s.g.

(VI) There is a free group $F$ and an epimorphism $\phi$ of $F$ onto $G$ such that $\operatorname{ker} \phi$ contains no nonzero pure subgroup of $F$.

Proof. (I) implies (II). Let $\phi: F \rightarrow G$ be a $q$-cover, $\left[z_{i} \mid i \in I\right]$ a basis of $F$ and $S=\left[\phi\left(z_{i}\right) \mid i \in I\right]$. If $S_{1}=\left[\phi\left(z_{i}\right) \mid i \in I^{\prime}\right]$ with $I^{\prime} \subset I$ and $I^{\prime}$ finite and if $\left\{S_{1}\right\}=\left\{c_{1}, \cdots, c_{m}\right\}$ with $m \leqq\left|I^{\prime}\right|$, then let $F_{1}=\left\{z_{i} \mid i \in I^{\prime}\right\}$ so that $\phi\left[F_{1}\right]=\left\{S_{1}\right\}$. Let $H$ be a free group of rank $m$ and $\sigma$ a homomorphism of $H$ onto $\left\{S_{1}\right\}$ which takes some basis of $H$ onto $\left[c_{1}, \cdots, c_{m}\right]$. If $\rho$ is a homomorphism of $F_{1}$ into $H$ such that $\rho \sigma$ $=\phi \mid F_{1}$, then $\operatorname{ker} \rho=0$ since $\operatorname{ker} \rho$ is a direct summand of $F_{1}$ and hence of $F$. Hence $\left|I^{\prime}\right| \leqq m$ so $m=\left|I^{\prime}\right|$.

(II) implies (III). Let $S$ be as in (II), $F$ a free group with basis $\left[z_{s} \mid s \in S\right]$ and let $\phi: F \rightarrow G$ be defined by $\phi\left(z_{s}\right)=s$. If $c=\sum r_{s} z_{s}$ is an element of $\operatorname{ker} \phi$ such that $F=\{c\}+H$, let $S_{1} \subset S$ be finite such that $r_{s}=0$ if $s \in S-S_{1}$ and let $F_{1}=\left\{z_{s} \mid s \in S_{1}\right\}$. Thus $F_{1}=\{c\}+F_{1} \cap H$. However, $\phi\left[F_{1}\right]=\phi\left[F_{1} \cap H\right]$ and $r\left(F_{1} \cap H\right)+1=r\left(F_{1}\right)=\left|S_{1}\right|$, that is, $\left\{S_{1}\right\}$ may be generated by fewer than $\left|S_{1}\right|$ elements of $G$ and (II) is contradicted.

(III) implies (IV). Let $\phi: F \rightarrow G$ be as in (III). If $\psi: F_{1} \rightarrow G$ is an epimorphism with $F_{1}$ free and if $\sigma: F \rightarrow F_{1}$ such that $\psi \sigma=\phi$ then ker $\sigma$ is a direct summand of $F$ and $\operatorname{ker} \sigma$ is contained in $\operatorname{ker} \phi$ so by (III) $\operatorname{ker} \sigma=0$ and $\sigma$ is a monomorphism.

(IV) implies (I). Let $\phi: F \rightarrow G$ be an $i^{*}$-cover of $G$. If $F=F_{2}+H$ and $\phi\left[F_{1}\right]=G$ then $\left\{F_{1}, \operatorname{ker} \phi\right\}=F$ and $\operatorname{ker} \phi=\operatorname{ker} \phi \cap F_{1}+L$ for some $L$. Hence $F=\left\{F_{1}\right.$, $\left.\operatorname{ker} \phi \cap F_{1}+L\right\}=F_{1}+L$. Let $\psi=\phi \mid F_{1}$ and let $\sigma$ be the projection of $F_{1}+L$ onto $F_{1}$, then $\psi \sigma=\phi$ so $\sigma$ is a monomorphism and $L=0$. It follows that $H=0$.

(II) is equivalent to (V). Let $S$ be as in (II) and suppose it is not a M.s.g. Then there is a subset $S_{1}$ of $S, S_{1}$ is finite, and a subset $T$ of $G$ with $|T|<\left|S_{1}\right|$ such that $G=\left\{\left(S-S_{1}\right), T\right\}$. Choose $S_{2} \subset S-\left(S_{1} \cup T\right)$ such that $S_{2}$ is finite and $S_{1} \subset\left\{T, S_{2}\right\}$; choose $S_{3} \subset S-\left(S_{1} \cup S_{2}\right)$ such that $S_{3}$ is finite and $T \subset\left\{S_{1}, S_{2}, S_{3}\right\}$. Since $S_{1} \cup S_{2} \subset\left\{T, S_{2}\right\}$ it follows that $\left\{T, S_{2}, S_{3}\right\}=\left\{S_{1}, S_{2}, S_{3}\right\}$. However, $\left|T \cup S_{2} \cup S_{3}\right| \leqq|T|+\left|S_{2}\right|$ $+\left|S_{3}\right|<\left|S_{1}\right|+\left|S_{2}\right|+\left|S_{3}\right|=\left|S_{1} \cup S_{2} \cup S_{3}\right|$. That is, $S_{1} \cup S_{2} \cup S_{3}$ is 
not a M.s.g. for $\left\{S_{1}, S_{2}, S_{3}\right\}$. This contradicts (II), hence (II) implies $(\mathrm{V})$. It is trivial that (V) implies (II).

(III) is equivalent to (VI). This follows from the fact that any pure subgroup of a free group contains a direct summand of the same rank $[1$, p. 194] and conversely any direct summand is pure.

This completes the proof of Theorem 1 . Notice that it has been shown that (II) can serve as a definition of M.s.g. Also, any $i^{*}$-cover is a $q$-cover and conversely. Finally if $S$ is a M.s.g. for $G$ then any free group with rank equal to $|S|$ together with any of the obvious epimorphisms forms a $q$-cover, and, conversely, if $\phi: F \rightarrow G$ is a $q$-cover then the image of any basis of $F$ is a M.s.g. for $G$.

Corollary 1. If $G$ is torsion free then $G$ has a M.s.g. iff $G$ is free.

Corollary 2. If $S$ and $T$ are M.s.g.'s of $G$ then $|S|=|T|$.

Corollary 3. If $r^{*}(G)$ is finite then $G$ has a M.s.g. iff $G$ is finitely generated.

Proof. If $r^{*}(G)=n$ then any finitely generated subgroup can be generated by fewer then $n+1$ elements, hence if $S$ is a M.s.g. $|S| \leqq n$.

Corollary 4. If $G$ has a M.s.g. then so does $G_{t}$.

Proof. If $\phi: F \rightarrow G$ is a $q$-cover, $\phi^{-1}\left[G_{t}\right]$ is pure in $F$.

Corollary 5. If $G$ has a M.s.g. and $G_{t}$ is small in $G$ then $G$ is free and hence $G_{t}=0$.

Proof. If $\phi: F \rightarrow G$ is a $q$-cover and $\eta: G \rightarrow G / G_{t}$ is the canonical epimorphism then $\eta \phi: F \rightarrow G / G_{t}$ is a $q$-cover of $G / G_{t}$. Hence $G / G_{t}$ is free and $G_{t}$ is a direct summand and $G_{t}=0$.

Corollary 6. If $G / G_{t}$ has a M.s.g. then $G$ splits (that is $G_{t}$ is a direct summand).

CoRollary 7. If $r^{*}(G)$ is finite and $G$ has a M.s.g. then $G$ splits.

In view of the last two corollaries it might be conjectured that if $G$ has a M.s.g. then $G$ splits. The following example shows that this is not the case.

ExAmple 1. Let $T=\sum_{i=1}^{\infty}\left\{a_{i}\right\}$ where the order of $a_{i}$ is $p^{2 i}$ (for a fixed prime $p$ ). Let $U=\prod_{i=1}^{\infty}\left\{a_{i}\right\}$ and let $b_{i} \in U$ be given by

$$
b_{i}=\left(0,0, \cdots, 0, a_{i}, p a_{i+1}, p^{2} a_{i+2}, \cdots\right) .
$$

If $H=\left\{T,\left[b_{i} \mid i=1,2, \cdots\right]\right\}$ then it is well known [1, p. 187] that $H$ does not split. However, $\left[b_{i} \mid i=1,2, \cdots\right]$ is a M.s.g. for $H$. It 
generates since $a_{i}=b_{i}-p b_{i+1}$. It is minimum since $\left\{b_{1}, \cdots, b_{n+1}\right\}$ $=\left\{a_{1}\right\}+\cdots+\left\{a_{n}\right\}+\left\{b_{n+1}\right\}$ so $\left\{b_{1}, \cdots, b_{n+1}\right\}$ cannot be generated by fewer than $n+1$ elements or else its reduced rank would be less than $n+1$ which is not the case [1, p. 52].

The next example shows that the converse of Corollary 6 is false even if it is assumed in addition that $G$ has a M.s.g.

Example 2. Let $\left(p_{0}, p_{1}, \cdots\right)$ be the set of primes, let

$$
Q^{\prime}=\left\{1 / p_{i} \mid i=0,1, \cdots\right\}
$$

and let $K$ be a direct sum of a countable number of cyclic groups of order $p_{0}$ with $e_{i}$ generating the $i$ th group $(i=1,2, \cdots)$. Let $G=Q^{\prime} \times K$ and

$$
S=\left[\left(1 / p_{i}, e_{i}\right) \mid i=1,2, \cdots\right] \cup\left[\left(1 / p_{0}, 0\right)\right] .
$$

Since each pair $\left(1 / p_{i}, 0\right)$ is in $\{S\}$ it follows that $Q^{\prime} \subset\{S\}$ and hence $K \subset\{S\}$ so $S$ generates $G$.

Let

$$
S^{\prime}=\left[\left(1 / p_{i}, e_{i}\right) \mid i=1, \cdots, n\right] \cup\left[\left(1 / p_{0}, 0\right)\right]
$$

and let $H=\left\{S^{\prime}\right\}$. Now, $Z \times\{0\} \subset H$ and $S^{\prime}$ is independent modulo $Z \times\{0\}$. Each pair $\left(1 / p_{i}, e_{i}\right)$ has order $p_{i} p_{0}$ modulo $Z \times\{0\}$ hence $r^{*}(H /(Z \times\{0\})) \geqq n+1$. It follows that $H /(Z \times\{0\})$ can be generated by no fewer than $n+1$ elements and hence neither can $H$. So $S^{\prime}$ is a M.s.g. for $H$. It follows immediately that $S$ is a M.s.g. for $G$.

Finally $G$ splits and $G / G_{t} \simeq Q^{\prime}$ with $r^{*}\left(Q^{\prime}\right)=1$ but $Q^{\prime}$ is not finitely generated and hence $G / G_{t}$ has no M.s.g.

According to Corollary 3 , if $G$ is not finitely generated but has a M.s.g. then $r^{*}(G)$ is infinite. On the other hand if $G_{i}$ is a direct sum of $i$ copies of $Z_{p_{i}}$ ( $p_{i}$ as in Example 2) and $G=\sum_{i=1}^{\infty} G_{i}$ then $G$ is not finitely generated and $r^{*}(G)$ is infinite but, by [3, Theorem 6], $G$ has no M.s.g.

If $\phi: F \rightarrow G$ and $\psi: F_{1} \rightarrow G$ are $q$-covers, then by (IV) $F$ and $F_{1}$ have the same rank and are thus isomorphic. However it is easy to construct an example such that there is no isomorphism $\rho$ such that $\psi \rho=\phi$.

Now if $\phi: G \rightarrow D$ is a monomorphism with $D$ divisible then $D$ is a divisible hull of $G$ iff for any monomorphism $\psi: G \rightarrow D_{1}$, with $D_{1}$ divisible, there is an epimorphism $\sigma: D_{1} \rightarrow D$ such that $\sigma \psi=\phi$. The following definition is a dual of this statement.

Definition. An $i$-cover of $G$ is a free group $F$ together with an epimorphism $\phi$ of $F$ onto $G$ such that, for any epimorphism $\psi$ of $F_{1}$ onto $G, F_{1}$ free, there is a monomorphism $\sigma$ of $F$ into $F_{1}$ such that $\psi \sigma=\phi$. 
Clearly any $q$-cover is an $i$-cover, however the following two theorems show that the converse is false.

Theorem 2. Any torsion free group has an i-cover.

Proof. If $G$ is free there is nothing to show so suppose $G$ is not free and in particular is not finitely generated.

Choose an epimorphism $\phi: F \rightarrow G$ with $F$ free and $r(\operatorname{ker} \phi)$ minimal among all such epimorphisms. It will be shown that $\phi: F \rightarrow G$ is an $i$-cover, so suppose $H$ is free and $\psi: H \rightarrow G$ is an epimorphism. Let $\sigma: F \rightarrow H$ be such that $\psi \sigma=\phi$ and it may as well be assumed that $\sigma[F]=H$.

There is a direct summand $K$ of $F$ such that ker $\phi \subset K$ and $r(K)$ $=r(\operatorname{ker} \phi)$. If $F=K+F_{1}$ then $F / K$ is isomorphic to $G / \phi[K]$ by the map induced by $\phi$. Since $F / K$ is free, $G=\phi[K]+G_{1}$ for some $G_{1}$ and $\phi \mid F_{1}$ is an isomorphism of $F_{1}$ onto $G_{1}$. Now ker $\sigma \subset K$ and $\operatorname{ker} \psi \subset \sigma[K]$ so that $H=\sigma[K]+\sigma\left[F_{1}\right]$ and $\psi \mid \sigma\left[F_{1}\right]$ and $\sigma \mid F_{1}$ are isomorphisms onto $G_{1}$ and $\sigma\left[F_{1}\right]$ respectively. The problem reduces to that of finding a commuting monomorphism of $K$ into $\sigma[K]$.

Now $r(\operatorname{ker} \psi) \leqq r(\sigma[K]) \leqq r(K)=r($ ker $\phi)$ so from the minimality of $r(\operatorname{ker} \phi)$ it follows that $r(\operatorname{ker} \psi)=r(\operatorname{ker} \phi)$.

If $K / \operatorname{ker} \phi$ is not of finite rank $|K / \operatorname{ker} \phi|=r(K / \operatorname{ker} \phi)$ and there is a free group $F^{\prime}$ with $r\left(F^{\prime}\right)=r(K / \operatorname{ker} \phi)$ and an epimorphism $\phi^{\prime}$ of $F^{\prime}+F_{1}$ onto $G$ with $\operatorname{ker} \phi^{\prime} \subset F^{\prime}$ so, since $r(K / \operatorname{ker} \phi) \leqq r(K)$, it follows that $r(K / \operatorname{ker} \phi)=r(K)$ or else $r\left(\operatorname{ker} \phi^{\prime}\right)<r(\operatorname{ker} \phi)$, contradicting the minimality of $r(\operatorname{ker} \phi)$. Since $\operatorname{ker} \phi$ is pure in $K, \operatorname{ker} \phi$ contains a direct summand $S$ of $K$ such that $r(S)=r(\operatorname{ker} \phi)$. So, in any case, $\operatorname{ker} \phi$ contains a summand $S$ of $K$ such that $r(S)=|K / \operatorname{ker} \phi|$. Similarly ker $\psi$ contains a direct summand $S^{\prime}$ of $\sigma[K]$ such that $r\left(S^{\prime}\right)=|\sigma[K] / \operatorname{ker} \psi|$.

Now $K / \operatorname{ker} \phi$ is isomorphic to $\sigma[K] / \operatorname{ker} \psi$ by the map induced by $\phi$ and $\psi, K$ and $\sigma[K]$ have infinite rank since ker $\phi$ and ker $\psi$ have infinite rank so $r(K)=r(\sigma[K]), \quad r(S)=|K / \operatorname{ker} \phi|$ and $r\left(S^{\prime}\right)$ $=|\sigma[K] / \operatorname{ker} \psi|$. It follows from a theorem of Erdös $[1$, p. 195] that there is an isomorphism $\theta$ of $K$ onto $\sigma[K]$ such that $\theta$ commutes with $\phi$ and $\psi$ on $K$ as desired.

THEOREM 3. If $G$ is not finitely generated and $G$ has the property that every epimorphism $\phi$ of a free group $F$ onto $G$ with $r(F)=|G|$ is such that $\operatorname{ker} \phi$ contains a direct summand of $F$ with rank equal to $r(F)$, then $G$ has an i-cover.

Proof. Let $F$ be free with $r(F)=|G|$ and let $\phi: F \rightarrow G$ be an epimorphism. It will be shown that this is an $i$-cover. 
Let $H$ be free and $\psi$ an epimorphism of $H$ onto $G$. It may be assumed that $r(H)=|G|$. According to the hypothesis both $\operatorname{ker} \phi$ and ker $\psi$ contain direct summands $S$ and $S_{1}$ of $F$ and $H$ respectively such that $r(S)=r\left(S_{1}\right)=r(F)=r(H)=|G|$. Moreover, since $r(S)=|F / \operatorname{ker} \phi|$ and $r\left(S_{1}\right)=|H / \operatorname{ker} \psi|$ and $F / \operatorname{ker} \phi$ is isomorphic to $H / \operatorname{ker} \psi$ by the map induced by $\phi$ and $\psi$, the theorem of Erdös cited above applies and $F$ is isomorphic to $H$ by a commuting isomorphism.

Corollary 8. Any countable group has an i-cover.

Proof. If $G$ has a $q$-cover then $G$ has an $i$-cover; if $G$ is countable and has no $q$-cover then Theorem 3 applies.

Remark. Among those groups with $i$-covers are those which have $q$-covers and those which very badly miss having $q$-covers. It should also be noted that in Theorem 2 and Theorem 3 an $i$-cover was very easy to get: all that was needed was a free group of the proper rank and an epimorphism. One might hope $G$ would have a quasi-projective cover (cf. [2]) iff $G$ has a $q$-cover. This is wishful thinking however since among the finitely generated, nontorsion free groups only the finite groups have quasi-projective covers.

\section{REFERENCES}

1. L. Fuchs, Abelian groups, Akad. Kiad6, Budapest, 1958; 3rd. ed., Pergamon Press, New York, 1960. MR 21 \#5672; MR 22 \#2644.

2. L. E. T. Wu and J. P. Jans, On quasi projectives, Illinois J. Math. 11 (1967), 439-448. MR 36 \#3817.

3. S. A. Khabbaz, Abelian torsion groups having a minimal system of generators, Trans. Amer. Math. Soc. 98 (1961), 527-538. MR 23 \#A3174.

University of South Carolina 